\title{
Establishment of A Comprehensive Service Management System for Retail Stores
}

\author{
Lu Qinghua \\ Tianjin Coastal Polytechnic, 300450
}

Keywords: Retail Store; Service Management; System; Comprehensive Service; Strategy

\begin{abstract}
Service management has always been one of the important tasks of retail enterprises, and has a greater impact on retail enterprises. Establishing a comprehensive service management system for retail stores can solve the dilemma faced by retail stores, clarify the customer base of the store, and thus improve the competitiveness of the store, thereby ensuring the image of the corporate brand. At present, the establishment of a comprehensive service management system for retail stores is not good, which affects the economic benefits of retail stores. Based on its existing problems, this paper analyzes the conditions that should be possessed by the comprehensive service management system, as well as the specific establishment methods, hoping to provide reference for relevant personnel.
\end{abstract}

\section{Introduction}

At present, retail stores have insufficient understanding of the comprehensive service management system, and have not formed clear management objectives, and cannot provide clear and high-quality services to consumers. The greater flexibility of consumers is not conducive to the expected economic benefits of retail stores. However, there are practical problems in it, some enterprises recognize the importance of comprehensive services. However, due to the limitations of the company's own strength or other factors, it is impossible to establish a comprehensive service management system. The quality of the service cannot always be improved, and the core competitiveness of retail stores has always been in a low state.

\section{Problems with Retail Store Services}

\subsection{Customer Perceived Service Issues}

The customers in retail stores are highly mobile, and they are not very concerned about the services provided by the stores. Even if the services provided by the various positions in the retail stores are biased, the customers themselves cannot form an objective evaluation of the services of the stores. Moreover, retail stores do not attach great importance to the establishment and provision of services, ignoring the basic demands of customers. The lack of integrity in the content of the service is an important cause of complaints from many customers. The retail store's understanding of retail services is still at an initial stage, and there is no recognition of the impact of services on the store. Online trading is a kind of service that is prosperous in China. Many stores have not provided it, which not only affects customer satisfaction, but also reduces revenue.

\subsection{Poor Service}

Retail stores tend to be small in size and do not pay enough attention to the setting of service items, so the service is not effective. Some of the larger retail stores will train service personnel in order to improve the quality of service. However, the deviation of specificity and purpose leads to the service personnel not having a better understanding of the training content. In practice, the services provided by these personnel still do not reach good service effect. Some retail stores do not pay attention to the improvement and optimization of service projects. They only provide a single service every year, which can not meet the needs of customers. The developed service projects are not based on actual conditions, and ultimately the service effect cannot be improved. For example, some retail stores will open a complaint channel in order to obtain customer support, but after the 
customer complains or comments, the handling of the complaint is not efficient.

\subsection{Service Response is Slow}

The customer's needs are immediacy, and the retail store's service response speed is slow and cannot meet the customer's specific needs. The project services of some stores have been in an unrenewed state for a long time, but the customer's needs are constantly changing, which makes the customer's needs unable to fully respond. Retail stores did not pass research and analysis when setting up service projects, resulting in their lack of obvious purpose in forming service projects, and could not exert actual results. For example, the service staff of a retail store negatively handles the problems reflected by the customer which delays the time, the wording is inappropriate or sloppy, that causes the consumer's patience is exhausted, and complaints about retail stores will increase.

\section{Establishment of A Comprehensive Service Management System for Retail Stores}

\subsection{Clear Service Object}

The establishment of service objects helps retail stores optimize service content and architecture, increase the purpose of services, and thus improve the quality of services. It is clear that the service target belongs to the foundation of the establishment of a comprehensive service management system for retail stores, which can deepen the impression that consumers treat brands. On this basis, retail stores can gradually promote brand personality and attract more customers. When a retail store builds a comprehensive service management system, the service object is studied as a focus, and the impact of the service object on the establishment of the service management system is analyzed. Based on this, we constantly adjust and optimize the style of store products, continuously research the current service status of retail stores, conduct research on the development of the brand and the future, and comprehensively analyze the significance of the establishment of service targets. Through such means, we can improve service quality, build a comprehensive service management system, enhance communication and communication between customers and service personnel, and let customers have better channels to express their own demands.

\subsection{Clear Service Standards}

Retail stores need to learn from good companies, master the current definition of services in the retail industry, and related service standards. The establishment of a comprehensive service management system can be achieved. Standardized and unified service standards are prerequisites for ensuring service quality. Retail companies also need to promote the formation of service standards to design specific service projects based on their own products and brand style. After the customer enters the store, the service personnel need to let the customer know the services and products provided in the store and get a good guide. If the customer does not feel uncomfortable in the process of being introduced to the service and enjoying the service, the customer will be considered as the first step to success. Retail stores start with customer needs and customer trials to meet customer requirements without affecting store operations and profits, thereby determining the most appropriate service method and ensuring healthy and orderly development of retail stores.

\subsection{Clear Service Standard Training Program}

In order to establish a comprehensive service management system, a service standard training plan needs to be clarified. In addition to the corresponding training programs, retail stores must also provide training for on-the-job employees in the store. The business training of retail store employees must develop a training plan in advance, and the funds, facilities and equipment required for employee training should be prepared in advance. In order to better cultivate a group of excellent employees, retail stores can organize employees to learn, and can also hire professional personnel for training. During the training period, the corresponding assessment targets should be set. Only the employees who meet the assessment criteria can enter the formal work position and control the service quality of the store in this way. The service ability of employees also needs to be 
improved and this is not done overnight. Instead, it is necessary to continuously carry out training and learning activities in practice, maintain regular training status, encourage employees to learn advanced knowledge and improve their professional level. These different measures can effectively carry out the establishment of a comprehensive service management system.

\subsection{Clear Service Management Performance Mechanism}

The performance mechanism is an important part of the comprehensive service management system. It can make employees work enthusiasm, stimulate their work enthusiasm, make them really responsible for the workers, constantly review their service behaviors, and ensure that the quality of services is greatly improved. The performance mechanism of retail stores can be an incentive mechanism to complete the screening of outstanding employees through the service quality assessment of employees. For example, the performance appraisal of sales personnel can be linked to performance, so that more employees can recognize the relationship between their service quality and their own income. Many employees' work abilities need to be tapped and motivated. Retail stores should gradually grasp the rules and provide employees with the same treatment as they do to ensure the employees' working status. The improvement of service quality depends on employees, so the performance mechanism built by retail stores needs to respect the opinions of employees and protect the interests of employees. When the performance mechanism is established and properly applied, the comprehensive service management system will be gradually improved, the quality of service provided by retail stores to customers will be improved, and the brand effect of retail stores will continue to increase in practice, making more economic profits for retail stores.

\section{Conclusion}

The establishment of a comprehensive service management system for retail stores requires certain conditions, and retail stores can be combined with actual conditions. In addition, comprehensive service management itself has certain overall characteristics, and its preventive effect is good. It belongs to retail stores and needs to have one management system. At present, the problems in the service effect, service system and quality of retail stores need to be solved urgently. It is necessary to construct a scientific and reasonable comprehensive service management system based on the characteristics of retail stores.

\section{References:}

[1] Wang Xiaohui. Analysis of the Status Quo of Space and Category of Network Retail Stores in Anhui Province[J].Journal of Honghe University,2018,16(05):101-104.

[2] Zheng Zhewen. Marketing model under the background of traditional retail store e-commerce [J]. Modern Business, 2018 (22): 32-33.

[3] Jiao Danlin. On the establishment of a comprehensive service management system for retail stores [J]. Modernization of shopping malls, 2017 (07): 12-13.

[4] Liu Haijuan, Ren Li. Analysis on the sales strategy of sports shoes brand retail stores [J]. Modern Decoration (theory), 2015 (10): 145.

[5] Long Yu. Research on omni-channel management of small and medium-sized retail stores based on Internet economy [J]. Shopping Mall Modernization, 2015 (23): 43.

[6] Lin Guangtao. How to establish a comprehensive service management system for terminal retail stores [J]. China Clothing, 2007 (02): 48-50. 